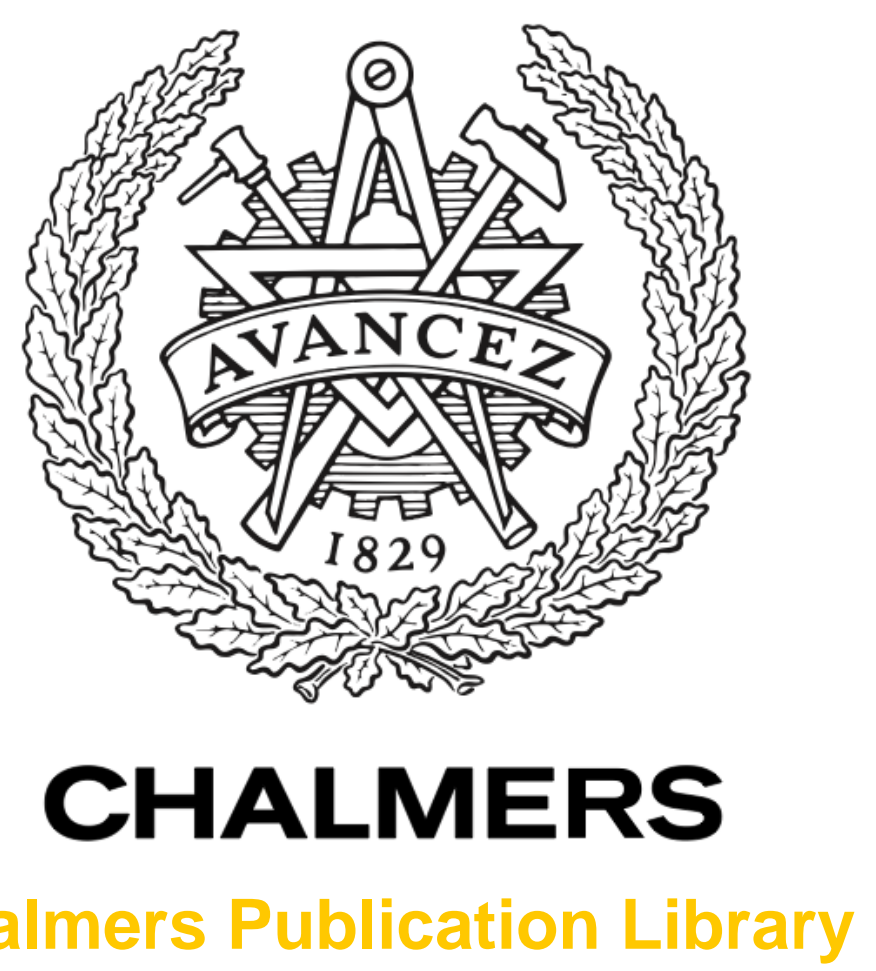

\title{
Short-block-length shaping by simple mark ratio controllers for granular and wide- range spectral efficiencies
}

This document has been downloaded from Chalmers Publication Library (CPL). It is the author's version of a work that was accepted for publication in:

\section{European Conference on Optical Communication (ECOC)}

Citation for the published paper:

Yoshida, T. ; Karlsson, M. ; Agrell, E. (2017) "Short-block-length shaping by simple mark ratio controllers for granular and wide-range spectral efficiencies". European Conference on Optical Communication (ECOC)

Downloaded from: http://publications.lib.chalmers.se/publication/254768

Notice: Changes introduced as a result of publishing processes such as copy-editing and formatting may not be reflected in this document. For a definitive version of this work, please refer to the published source. Please note that access to the published version might require a subscription. 


\title{
Short-Block-Length Shaping by Simple Mark Ratio Controllers for Granular and Wide-Range Spectral Efficiencies
}

\author{
Tsuyoshi Yoshida(1,2), Magnus Karlsson(1), and Erik Agrell(1) \\ (1) Chalmers University of Technology, SE412-96 Gothenburg, Sweden, tsuyoshi@chalmers.se \\ (2) Mitsubishi Electric Corporation, 5-1-1 Ofuna, Kamakura, Kanagawa, 247-8501 Japan, \\ Yoshida.Tsuyoshi@ah.MitsubishiElectric.co.jp
}

\begin{abstract}
We propose a simple mark ratio controller for probabilistic shaping. It realizes granular spectral efficiencies from 1 to $9 \mathrm{~b} / \mathrm{s} / \mathrm{Hz} /$ polarization and $0.2 \mathrm{~dB}$ lower required SNR than CCDM under a much shorter block length of 64 .
\end{abstract}

\section{Introduction}

Shaping is currently receiving much attention as a way to approach the Shannon capacity ${ }^{1-5}$. Böcherer et al. have proposed probabilistically amplitude shaping (PAS) ${ }^{1}$, which outperforms uniform signalling at the expense of more complex hardware of a distribution matcher and a dematcher. The implementation of them is a big issue because strong shaping requires large block lengths ${ }^{1-4}$ such as 1,000 or 10,000 symbols for around $1 \mathrm{~dB}$ required signal-to-noise ratio (SNR) reduction. Böcherer et al. further proposed product distribution matching for simpler implementation by combining binary distribution matchers and natural coding ${ }^{3}$. However, the block length issue remains. Under a limited block length condition, storing the best code sets in a look-up table (LUT) ${ }^{5}$ might be an option, but it is complex and consumes much hardware resources unless further simplification is done, which is yet to be shown 5 .

In this paper, we propose a simple mark ratio controller (MRC) and demonstrates its application to product distribution matching. The block length of the shaping, $N_{\mathrm{s}}$, is limited to 64 . The matching scheme enables granular spectral efficiencies over a wide range, from 1 to 9 $\mathrm{b} / \mathrm{s} / \mathrm{Hz} /$ pol., and achieves typically $0.2 \mathrm{~dB}$ lower required SNR than PAS with constant composite distribution matching (CCDM) under the same $N_{s}$ of 64 , without complex operations ${ }^{1-4}$ or a full LUT5. $^{5}$.

\section{Principle of the shaping scheme}

The signal flow is shown in Figure 1, with the configuration of the transmitter and receiver in Fig. 1 (a). The input data bits are demultiplexed to a vector $\boldsymbol{A}$ and shaped by the distribution matcher into a vector $\boldsymbol{B}$. The vector sizes are $m$, which is the number of bit tributaries for the pulse amplitude modulation (PAM) symbol. It is followed by a systematic binary forward error correction (FEC) encoder and PAM mapper with a binary reflected Gray code (BRGC) to generate
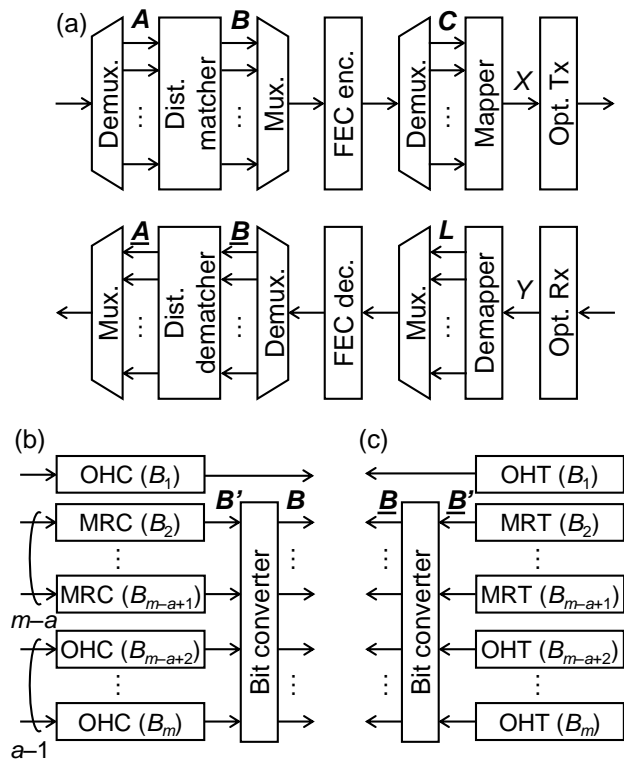

(c)

Fig. 1: Signal flow of the shaping scheme.

(a) Full configuration of TRx, (b) distribution matcher, and (c) distribution dematcher.

the symbol $X$. After the optical channel, the received signal is coherently detected and demodulated into the symbol $Y$, which is demapped into a bitwise log-likelihood ratio (LLR) vector $\boldsymbol{L}$. The LLRs are multiplexed, decoded, and demultiplexed again to obtain the vector $\underline{\boldsymbol{B}}$. The shaping is terminated in the distribution dematcher to recover the original data bits as the received vector $\underline{\boldsymbol{A}}$.

The distribution matcher shown in Fig. 1 (b) consists of a mark ratio controller (MRC) for each bit tributary, and a bit converter. We propose two types of MRCs. The first one is a bit scramble selector shown in Fig. 2. The input bit sequence having a block length $N_{s}$ is divided into $2^{N \text { scr lanes }}$ and each of them is XOR:ed with an unique scrambling sequence; e.g. an arbitrary shifted pseudo-random binary sequence. We compare the mark count $n_{\mathrm{m}}(i)$ of each scrambled sequence $i$, and select the sequence $(i s)$ that maximizes $\left|n_{\mathrm{m}}(I)-N_{\mathrm{s}} / 2\right|$. If $n_{\mathrm{m}}\left(i_{\mathrm{s}}\right)$ is larger than $N_{\mathrm{s}} / 2$, the sequence is inverted. The sequence selection and inversion control can be described by $N_{\text {scr }}$ 
bits and 1 bit, respectively. We carry the $N_{\text {scr }}+1$ bits as explicit overhead on the specific bit tributary (shown as $\mathrm{OHC}$, overhead controller). It is terminated in the overhead terminator $(\mathrm{OHT})$ and utilized in the mark ratio control terminator (MRT) at the distribution dematcher. Though the explicit overhead is usually carried on the sign-bit of the PAM symbol following the PAS concept ${ }^{1}$, we generalize PAS to place these overhead bits on amplitude bits of the PAM symbols, too, as will be explained later.

The second MRC is a permutation mapper with block length $N_{s}$. The codebook consists of the all-zero sequence, all $N_{\mathrm{s}}$ single-mark sequences, and $N_{s}^{2} / 2-N_{s}-1$ of the $N_{s}^{2} / 2-N_{s} / 2$ possible double-mark sequences. These $N_{s}^{2} / 2$ sequences can together carry $2 \log _{2} N_{s}-1$ bits of information. An example of a codebook for $N_{s}=4$ (3 bit input, 4 bit slot output) is shown in Tab. 1 . The top half of the codebook consists of single mark and the bottom half does double marks. The number of baseline sequences is 1 for single mark and $\left(N_{\mathrm{s}} / 2-1\right)$ for double mark, and the number of circular shift pattern is $N_{s}$ for both cases. When there is an all mark input, an all zero codeword is provided. The permutation mapper does not have explicit overhead.

The bit converter after MRCs for non-sign-bits performs natural-to-BRGC conversion. The resulting probabilities become

$$
P_{|X|}(x)=\prod_{i=2}^{m} P_{B_{i}^{\prime}}\left(\bmod \left(\left\lfloor\frac{x-1}{2^{m-i+1}}\right\rfloor, 2\right)\right) .
$$

An example for 16-PAM is shown in Tab. 2. This will be terminated at the dematcher with its

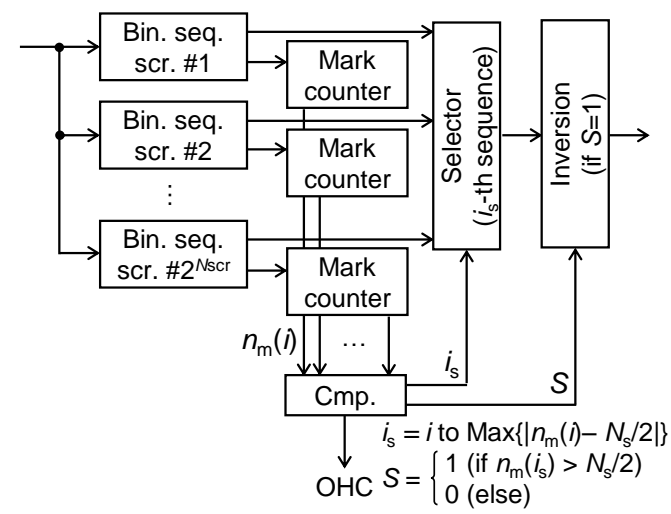

Fig. 2: Bit scramble selector as a mark ratio controller.

Tab. 1: Example of a permutation mapper.

\begin{tabular}{|c|c|}
\hline Input & Output \\
\hline 000 & 0001 \\
\hline 001 & 0010 \\
\hline 010 & 0100 \\
\hline 011 & 1000 \\
\hline 100 & 0011 \\
\hline 101 & 0110 \\
\hline 110 & 1100 \\
\hline 111 & 0000 \\
\hline
\end{tabular}

Tab. 2: Bits converter for 16-PAM

\begin{tabular}{|c|c|c|}
\hline$B_{2}{ }^{\prime} B_{3}{ }^{\prime} B_{4}{ }^{\prime}$ & $B_{2} B_{3} B_{4}$ & $|X|$ \\
\hline 000 & 000 & 1 \\
\hline 001 & 001 & 3 \\
\hline 010 & 011 & 5 \\
\hline 011 & 010 & 7 \\
\hline 100 & 110 & 9 \\
\hline 101 & 111 & 11 \\
\hline 110 & 101 & 13 \\
\hline 111 & 100 & 15 \\
\hline
\end{tabular}

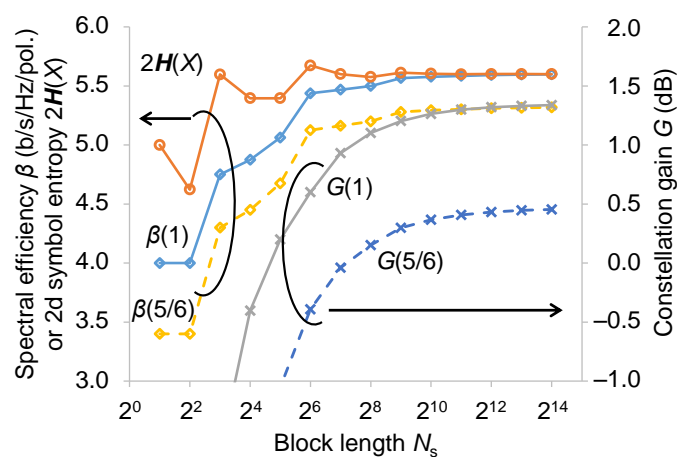

Fig. 3: Shaping and modulation spectral efficiency $\beta, 2 d$ symbol entropy $2 \boldsymbol{H}(X)$, or constellation gain $G$ as a function of block length $N_{s}$.

inverse function.

Based on PAS, we should place non-shaped bits such as the FEC parity bits on the sign-bit of the PAM symbol not to degrade the amplitude shaping characteristics. In this paper we generalize the PAS to allocate the overheads to a-1 amplitude bits. The relationship between shaping rate $R_{\mathrm{s}}$ and FEC code rate $R_{\mathrm{c}}$ is

$$
\begin{gathered}
R_{\mathrm{s}}=\frac{N_{\mathrm{f}}+N_{\mathrm{v}}}{(m-a) N_{\mathrm{s}}+N_{\mathrm{eOH}}+N_{\mathrm{v}}}, \\
N_{\mathrm{v}}=a N_{\mathrm{s}}-N_{\mathrm{eOH}}-\left(1-R_{\mathrm{c}}\right) m N_{\mathrm{s}},
\end{gathered}
$$

where $N_{\mathrm{f}}, N_{\mathrm{v}}$, and $N_{\mathrm{eOH}}$ are number of fixed information bits, variable information bits depending on $R_{\mathrm{c}}$, and explicit shaping overhead per $N_{\mathrm{s}}$ symbols. From (3), the minimum $R_{\mathrm{c}}$ is

$$
R_{\mathrm{c}, \min }=\left((m-a) N_{\mathrm{s}}+N_{\mathrm{eOH}}\right) /\left(m N_{\mathrm{s}}\right) \text {. }
$$

While the original PAS $(a=1)$ tightly limits $R_{\mathrm{c}}$, the parameter $a=2$ will significantly enlarge the flexibility in the choice of $R_{\mathrm{c}}$. The least significant bit of the PAM symbol is suitable for using as the overhead carrier. When we shape the signal with shaping rate $R_{\mathrm{s}}$, the shaping and modulation spectral efficiency $\beta$ [b/s/Hz/pol.] becomes $2 m R$ s.

\section{Block length dependency}

We now show the importance of the block length. The state-of-the-art CCDM realizes quite efficient bits-to-bits conversion with sufficiently large block lengths ${ }^{2}$. The target probability mass function $\left[P_{|X|}(1), P_{|X|}(3), P_{|X|}(5), P_{|X|}(7)\right]$ was set to [0.6112, $0.3042,0.0754,0.0092]$. The output probability mass function was quantized according to the output block length $N_{\text {s. }}$. The $\beta, 2$ dimensional (2d) symbol entropy $2 \boldsymbol{H}(X)$, and constellation gain 6 $G=\left(2^{\beta}-1\right) d_{\min ^{2}} /(6 E)$ are shown in Fig. 4, where $d_{\text {min }}$ is minimum Euclidean distance and $E$ is average energy in $2 \mathrm{~d}$. $R_{\mathrm{c}}$ was assumed to be 1 or $5 / 6$. The corresponding $\beta$ and $G$ are shown as $\beta\left(R_{\mathrm{c}}\right)$ or $G\left(R_{\mathrm{c}}\right)$. The $2 \boldsymbol{H}(X)$ does not give the true rate, and $\beta$ decreases as $N_{\mathrm{s}}$ decreases. Though the acceptable value of $N_{\mathrm{s}}$ has not been much studied yet, $2^{6}$ would be manageable for such function. While $\beta(1)$ is $4.598 \mathrm{~b} / \mathrm{s} / \mathrm{Hz} / \mathrm{pol}$. and $G(1)$ is $1.34 \mathrm{~dB}$ under the nearly ideal condition of 
$N_{s}=2^{14}, \beta(1)$ is $96.5 \%$ and $G(1)$ is limited to $0.6 \mathrm{~dB}$ if $N_{s}=2^{6} . \beta(5 / 6)$ and $G(5 / 6)$ are further less than $\beta(1)$ and $G(1)$.

\section{Basic figure of merits}

We calculated constellation gain and spectral efficiency to extract good combinations of the considered low-complexity matchers based on 4 , 8, 16, and 32-PAMs. There are 24 different MRCs and they are used in bit tributaries $B_{2}{ }^{\prime}$ to $B_{5}{ }^{\prime}$ independently. Though only the useful MRCs and the combination are shown in Tabs. 3 and 4 due to space limitations, we examined $24^{m-1}$ combinations for $2^{m}$-PAM. Figure 4 shows $G(1)$ as a function of $\beta(1)$. Dips in $G(1)$ are observed at $\beta(1)=3,5,7$, and $9 \mathrm{~b} / \mathrm{s} / \mathrm{Hz} /$ pol. The minimum $G(1)$ is around $0.4 \mathrm{~dB}$ over 3.5 to $8.8 \mathrm{~b} / \mathrm{s} / \mathrm{Hz} / \mathrm{pol}$. If we employ 64-PAM, the dip at 8.8 to $9 \mathrm{~b} / \mathrm{s} / \mathrm{Hz}$ will be less pronounced. When some gap of $\beta(1)$ is acceptable, this technique can cover a quite wide range from 2 to $10 \mathrm{~b} / \mathrm{s} / \mathrm{Hz} / \mathrm{pol}$. with reasonable complexity.

\section{Simulations}

We simulated the post-FEC performance over the Gaussian channel. The DVB-S2 low density parity check $\operatorname{codes}^{7}$ were utilized, having the code length of 64800 and the tested code rates $R_{c}$ were $2 / 3,3 / 4,5 / 6$, and $9 / 10$. The number of decoder iterations was 20 . The tested combinations of MRCs were the same as the list in Tab. 4. For the comparison, 64-QAMs shaped by CCDM with limited block lengths $N_{\mathrm{s}}$ of 64 and 1024 were simulated. The target $2 \boldsymbol{H}(X)$ was set to $4.13,4.60,5.23$, and 5.73 bits, taken from Ref $^{4}$. Uniform signaling of QPSK, 8, 16, 32, 64, 256, and 1024-QAM were examined, too. The required SNR to obtain a post-FEC bit error rate less than $10^{-3}$ was evaluated.

Figure 5 shows the SNR gap from the Shannon limit vs. spectral efficiency $\left(\eta=R_{c} \beta\right)$. If we use CCDM with sufficiently large block length ${ }^{3}$ such as $N_{\mathrm{s}}=1024$, the SNR gap is reduced by 0.5 to $1 \mathrm{~dB}$ compared to uniform signals. In the more practical case of $N_{s}=64$, the benefit from CCDM is decreased to $\sim 0.2 \mathrm{~dB}$. The proposed MRC having $N_{\mathrm{s}}$ at most 64 realizes $\sim 0.2 \mathrm{~dB}$ better performance than CCDM over 1 to $9 \mathrm{~b} / \mathrm{s} / \mathrm{Hz} / \mathrm{pol}$. combining with rate adaptive FEC without complex operations ${ }^{1-4}$ or full set of LUT $^{5}$ for shaping.

\section{Conclusion}

We proposed a much shorter block length distribution matching. Compared to CCDM with block length of 64 , it realizes around $0.2 \mathrm{~dB}$ better performance over 1 to $9 \mathrm{~b} / \mathrm{s} / \mathrm{Hz} / \mathrm{pol}$.

\section{References}

[1] G. Böcherer et al., "Bandwidth efficient and ..." IEEE
Tab. 3: Parameters of mark ratio controllers.

\begin{tabular}{|c|c|c|c|c|c|}
\hline$\#$ & Type & Mark ratio & $N_{\mathrm{f}}$ & $N_{\mathrm{eOH}}$ & $N_{\mathrm{s}}$ \\
\hline 1 & Uniform & 0.5 & 1 & 0 & 1 \\
\hline 2 & Zero & 0 & 0 & 0 & 1 \\
\hline 3 & Bit scr. & 0.327 & 32 & 4 & 32 \\
\hline 4 & Bit scr. & 0.450 & 64 & 1 & 64 \\
\hline 5 & Bit scr. & 0.421 & 64 & 2 & 64 \\
\hline 6 & Bit scr. & 0.377 & 64 & 4 & 64 \\
\hline 7 & Perm. & 0.116 & 7 & 0 & 16 \\
\hline 8 & Perm. & $6.04 \mathrm{E}-2$ & 9 & 0 & 32 \\
\hline 9 & Perm. & $3.07 \mathrm{E}-2$ & 11 & 0 & 64 \\
\hline
\end{tabular}

Tab. 4: Relevant combination of mark ratio controllers.

\begin{tabular}{|c|c|c|c|c|c|c|}
\hline \multicolumn{3}{|c|}{ MRC combination } & \multicolumn{3}{c|}{ Figure of merits } \\
\hline$B_{2}{ }^{\prime}$ & $B_{3}{ }^{\prime}$ & $B_{4}{ }^{\prime}$ & $B_{5}{ }^{\prime}$ & $N_{\mathrm{s}}$ & $\begin{array}{c}\beta(1) \\
\text { (b/s/Hz/pol. })\end{array}$ & $\begin{array}{c}G(1) \\
(\mathrm{dB})\end{array}$ \\
\hline$\# 9$ & $\# 2$ & $\# 2$ & $\# 2$ & 64 & 2.344 & 0.424 \\
\hline$\# 9$ & $\# 6$ & $\# 2$ & $\# 2$ & 64 & 4.219 & 0.745 \\
\hline$\# 3$ & $\# 6$ & $\# 2$ & $\# 2$ & 64 & 5.625 & 0.654 \\
\hline$\# 8$ & $\# 6$ & $\# 4$ & $\# 2$ & 64 & 6.406 & 0.740 \\
\hline$\# 3$ & $\# 6$ & $\# 4$ & $\# 2$ & 64 & 7.593 & 0.644 \\
\hline$\# 7$ & $\# 6$ & $\# 4$ & $\# 1$ & 64 & 8.719 & 0.611 \\
\hline$\# 3$ & $\# 5$ & $\# 4$ & $\# 1$ & 64 & 9.653 & 0.658 \\
\hline
\end{tabular}

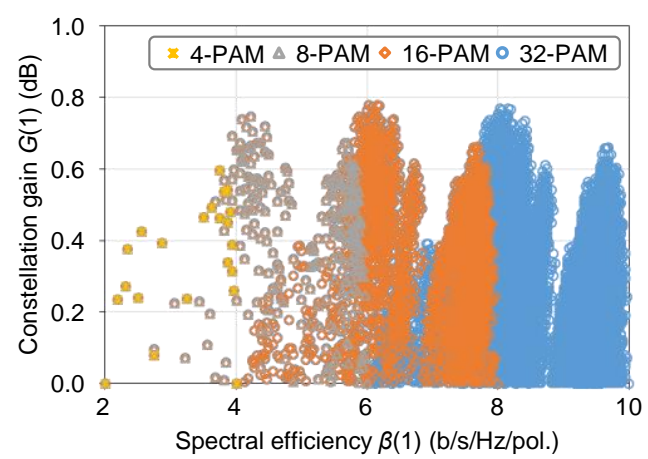

Fig. 4: Constellation gain $G(1)$ as a function of shaping and shaping and modulation spectral efficiency $\beta(1)$.

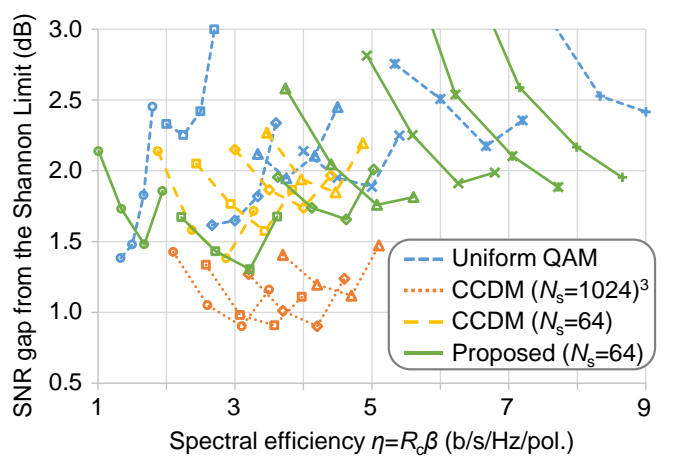

Fig. 5: The simulated SNR gap from the Shannon Limit as a function of total spectral efficiency $\left(\eta=R_{c} \beta\right)$.

Trans. Commun., vol. 63, no. 12, p. 4651 (2015).

[2] P. Schulte et al., IEEE Trans. Inf. Theory, "Constant composition distribution ...," vol. 62, no. 1, p. 430 (2016).

[3] G. Böcherer et al., "High throughput probability ...," Preprint, arXiv:1702.07510v1 (2017).

[4] F. Buchali et al., "Rate adaptation and ...," J. Lightw. Technol., vol. 34, no. 7, p. 1599 (2016).

[5] S. Chandrasekhar et al., "High-spectral-efficiency ...," in Proc. ECOC, paper Th.3.C.1 (2016).

[6] F. Kschischang et al., "Optimal nonuniform signaling ...," IEEE Trans. Inf. Theory, vol. 39, no. 3, p. 913 (1993).

[7] ETSI, "Digital video broadcasting ...," EN 302 307-1, v.1.4.1 (2014). 